\title{
Nouvelles cartes géographiques
}

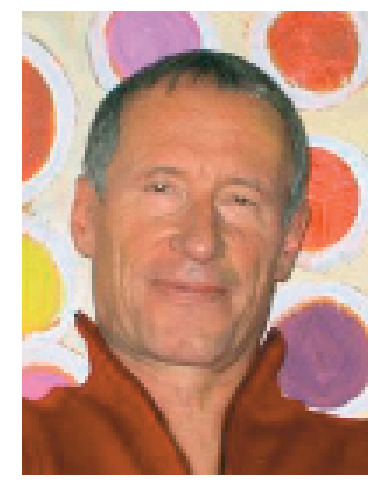

- du. Zeitschrift für Kultur. Weltkarten. Eine Vermessenheit. Nr. 11/12, Dezember 2005, Januar 2006.

- Kehlmann D. Die Vermessung der Welt. Reinbek: Rowohlt; 2005. 302 Seiten.
Sans carte, on ne s'y retrouve pas. Elles permettent de s'orienter aussi sur des lieux que nous n'atteindrons jamais: la face cachée de la lune ou le fond des océans. Les cartes sont des images complexes extrêmement transformables qui rendent compte d'un relevé plus ou moins réaliste de la surface de la terre. Les quatre architectes Jacques Herzog, Christian Schmid, Marcel Meili et Roger Diener nous en donnent un exemple actuel avec leurs cartes suisses qui ont mis en émoi à la fin de l'année dernière. Les «faiseurs de suisses» (Schweizermacher), comme on les a nommés dans le Tages-Anzeiger, tirent le portrait urbanistique d'un pays citadin. Leurs nouvelles visions conduisent à d'inhabituels échelonnements de courants pendulaires, réseaux de villes, régions métropolitaines, zones calmes, contrées alpines et à ce qu'ils appellent de manière provocante jachère alpine. Comme quoi, dans l'histoire de la cartographie, chaque nouvel étalon ouvre de nouveaux horizons. Les limites traditionnelles disparaissent et rendent visibles les nouvelles zones de conflit, régions reculées et potentiels de développement de notre pays. Par la médecine, nous sommes habitués à des représentations qui projettent les données épidémiologiques sur les cartes géographiques. Si nous transposions toutes les données démographiques et statistiques médicales proportionnellement, le résultat serait de nouvelles topographies inhabituelles de la santé. Les domaines de souveraineté politiquement essentiels n'y paraîtraient plus, ce qui aurait sûrement des conséquences. Agir selon des coordonnées inhabituelles pourvoit toujours à des discernements surprenants. En effet, même au siècle des systèmes de navigation régis par satellites, la détermination de la position reste un problème, pas seulement sur le plan territorial.

Mais pour cela il existe heureusement des guides compétents, par exemple la revue «du» de décembre 2005, janvier 2006. Elle porte le titre «cartes du monde. Une conjecture.» On y trouve presque tout ce qu'on devrait savoir sur l'histoire, l'aspect et les problèmes des cartes: sur les cartes historiques de la Babylone sumérienne jusqu'à la géologie du Cervin, de la plaque eurasienne à la dérive des continents d'Alfred Wegener, des dégâts de l'agent orange au Vietnam aux flux de marchandises d'aujourd'hui. Chaque représentation rend compte d'une manière de penser, d'une nouvelle connaissance ou d'une vision du monde particulière, que les surfaces soient représentées selon la grandeur du produit social brut de 1996, qu'il s'agisse d'un atlas coréen de 1700 après J.-C. qui représente l'Empire du Milieu avec un espace limitrophe de barbares, géants cyclopéens et mythiques amazones. Des exemples d'images suggestives, des commentaires remarquables et des considérations avisées de divers auteurs entraînent le lecteur sur le chemin des découvertes. De Daniel Kehlmann, né en 1975, on peut lire l'article sur «celui qui voyage avec le doigt, le conquistador de la fantaisie qui, souvent myope, voyage timidement et rêve avec le doigt à travers les continents lointains».

Il a aussi écrit un livre qu'on peut recommander à tous ceux qui aimeraient approfondir le thème des cartes et des voyages d'une manière distrayante: «L'arpentage du monde». Un roman palpitant qui décrit la vie de deux génies en scènes réelles et fictives. L'un, Alexandre de Humboldt (1769-1859), atteint une renommée mondiale avec ses collections et ses descriptions de voyages. Il ne recule devant aucune peine et ne craint aucun tabou quand il s'agit d'arpenter et de cataloguer les montagnes géantes et les forêts vierges avec son compagnon Bonpland. L'autre, Carl Friedrich Gauss (1777-1855) n'a pratiquement jamais quitté son lieu d'origine et conquiert pourtant la terre entière avec ses formules mathématiques et l'astronomie. «L'arpentage du monde» est un jeu raffiné, une biographie divertissante et pleine d'humour mais aussi une histoire d'aventure philosophique sur deux remarquables architectes de la modernité, dont chaque enfant d'âge scolaire connaît aujourd'hui le nom.

Erhard Taverna 